'Servicio de Gastroenterología Hospital Austral. Buenos Aires, Argentina.

${ }^{2}$ Servicio de Gastroenterología

Clínica Las Condes. Santiago, Chile.

3Programa Enfermedad Inflamatoria Intestinal. Clínica Las Condes. Santiago, Chile.

${ }^{4}$ Unidad Coloproctología Clínica

Las Condes. Santiago, Chile.

${ }^{5}$ Dirección Académica, Clínica Las

Condes. Santiago, Chile.

aBecada de Gastroenterología.

bEnfermera.

Los autores declaran no tener conflictos de interés. Este trabajo no recibió financiamiento.

Recibido el 28 de febrero de 2018, aceptado el 18 de diciembre de 2018.

Correspondencia a: Dr. Rodrigo Quera

Clínica Las Condes. Estoril 450, Las Condes. Santiago, Chile. Teléfono 226108840. rquera@clc.cl

\section{Factores ambientales en el desarrollo y evolución de la enfermedad inflamatoria intestinal}

\author{
NOELIA E. MELIGRANA ${ }^{1, a}$, RODRIGO QUERA ${ }^{2,3}$, \\ CAROLINA FIGUEROA ${ }^{2,3}$, PATRICIO IBÁÑEZ ${ }^{2,3}$, JAIME LUBASCHER ${ }^{2,3}$, \\ UDO KRONBERG ${ }^{3,4}$, LILIAN FLORES ${ }^{2,3, b}$, DANIELA SIMIAN $^{3,5, b}$
}

\section{Environmental risk factors in the development and evolution of inflammatory bowel disease}

Environmental factors may influence the development of Inflammatory Bowel Disease and modify its natural history. The objective of this review is to evaluate current evidence about environmental factors associated with the disease. A better knowledge about the pathogenesis of the disease can lead to better treatment strategies and suggestions to prevent the disease.

(Rev Med Chile 2019; 147: 212-220)

Key words: Colitis, Ulcerative; Crohn Disease; Environment; Inflammatory Bowel Diseases; Pathology.
L a enfermedad inflamatoria intestinal (EII), que incluye la colitis ulcerosa $(\mathrm{CU})$ y la enfermedad de Crohn (EC), son enfermedades crónicas de causa desconocida que se desarrollan en personas genéticamente predispuestas sobre la que interactúan factores ambientales, la microbiota y una desregulación del sistema inmune $^{1}$. Su incidencia ha ido en aumento en países desarrollados, y estudios en países en vías de desarrollo de Sudamérica, África y Asia han demostrado también un aumento significativo de la EII ${ }^{2}$. Aunque la incidencia y prevalencia en Chile aún es desconocida, un estudio ha sugerido un aumento de estas enfermedades ${ }^{3}$. En las últimas décadas, la EII ha surgido como un desafío de la salud pública, debido al aumento de incidencia, a la afectación principalmente del adulto joven y a los costos de su tratamiento ${ }^{2,4}$. Es posible especular que este cambio epidemiológico sea producto de la interacción entre factores genéticos y ambientales con la microbiota. Estos últimos han sido definidos bajo el término de exposomas, el cual incluye todos los factores ambientales que se pueden presentar desde el nacimiento hasta la muerte afectando la evolución de diferentes enfermedades, incluyendo la EII 5 .

El objetivo de esta revisión es describir aquellos factores ambientales que pueden influir en el desarrollo y evolución de la EII (Tabla 1). Conocerlos mejor abre una oportunidad para nuevas estrategias de tratamiento y prevención de la enfermedad.

\section{Factores asociados a la protección contra la EII}

\section{Lactancia materna}

La leche materna aporta antígenos microbianos e inmunoglobulinas que favorecen la tolerancia inmune, transferencia de anticuerpos maternos y mayor diversidad de la microbiota ${ }^{6}$. Un metaanálisis demostró que la lactancia materna, de cualquier duración, es un factor protector en pacientes con EC y CU (OR 0,45; intervalo de confianza $95 \%$ (IC95\%) 0,26-0,79 y OR 0,56; IC95\% 0,38-0,8, respectivamente) ${ }^{7}$. La ausencia 
Tabla 1. Factores ambientales que influyen en el desarrollo y evolución de la enfermedad inflamatoria intestinal

\begin{tabular}{|lll|}
\hline Factores protectores & Factores perjudiciales & Factores paradojales \\
\hline Lactancia materna & Calidad del sueño & Dieta (PUFA) \\
\hline Infección por Helicobacter pylori & Polución/urbanización & Apendicectomía \\
\hline Probióticos/prebióticos & Obesidad & Tabaco \\
\hline Ejercicio físico & Antiinflamatorios no esteroidales (AINE) \\
\hline Vitamina D & Antibióticos/gastroenteritis infecciosa \\
\hline & Anticonceptivos orales \\
\hline & Hipoxia/altura $>2.000$ metros sobre nivel del mar \\
\hline
\end{tabular}

PUFA: ácidos grasos poliinsaturados.

o duración de la lactancia materna menor a 3-6 meses ha sido considerada un factor de riesgo para el desarrollo de EII ${ }^{8}$.

\section{Helicobacter pylori}

La infección por Helicobacter pylori está inversamente relacionada con la incidencia de EII (OR 0,43; IC95\% 0,36-0,5) . Mientras que el mecanismo subyacente aún no se ha explorado, Helicobacter pylori conduce a un aumento de la expresión de Foxp3 en la mucosa, el factor de transcripción de las células $\mathrm{T}$ reguladoras, que podría explicar este efecto protector mediante la regulación negativa del proceso inflamatorio ${ }^{10}$.

\section{Probióticos/Prebióticos}

Los probióticos modulan la composición de la microbiota inhibiendo el crecimiento de bacterias potencialmente patógenas a través de la producción de bacteriocinas y la creación de un medio más ácido que es hostil para las bacterias proinflamatorias, también promueven el crecimiento de especies beneficiosas como lactobacilos y bifidobacterias ${ }^{11}$. El más destacado es el Bifidobacterium longum spp longum 35624, debido a sus efectos antiinflamatorios ${ }^{12}$. El impacto de los probióticos en la EII ha sido objeto de varias revisiones sistemáticas y metaanálisis. En CU, los lactobacilos han demostrado atenuar el daño histológico y conducir en un porcentaje importante a la remisión ${ }^{13}$. En un metaanálisis se demostró que los probióticos aumentan significativamente las tasas de remisión en pacientes con CU activa
(RR 1,51; $\mathrm{p}=0,01)^{14}$. El análisis de subgrupos encontró que solo VSL\#3 (mezcla de cepas de Bifidobacterium, Lactobacillus y Streptococcus salivarius spp. termophilus) aumentó las tasas de remisión en comparación con los controles en pacientes con CU activa (RR 1,$74 ;$ p: 0,004$)$ y mantuvo la remisión en pacientes con reservoritis (RR 0,$18 ; \mathrm{p}<0,0001)^{15}$. La combinación de probióticos con 5-aminosalicilatos (5-ASA) es superior a 5-ASA solo en la inducción y mantenimiento de la remisión en CU. En cambio, los probióticos no son superiores al placebo para la inducción a la remisión y mantenimiento en la $\mathrm{EC}^{14}$. A pesar de la importancia de la microbiota y de los resultados referidos, creemos que aún faltan estudios que sustenten mejor la efectividad real de esta estrategia terapéutica en el manejo de la EII.

Los prebióticos se definen como carbohidratos no digeribles que promueven selectivamente la actividad y el crecimiento de una o más cepas de bacterias benéficas en el intestino grueso, dentro de los cuales se encuentran inulina, fructooligosacárido, galactooligosacárido y lactulosa ${ }^{16}$. Estos son utilizados por bacterias y metabolizados a ácidos grasos de cadena corta estimulando la biosíntesis de mucina, mejorando la barrera intestinal e inhibiendo la adhesión de microorganismos patógenos a las células intestinales ${ }^{16}$. Aunque existe escasa evidencia para sugerir el uso de prebióticos en EII, el efecto de la microbiota en el desarrollo y evolución de la enfermedad plantea la necesidad de estudios de mayor calidad que permitan definir su rol como tratamiento ${ }^{17}$. 


\section{Ejercicio físico}

La actividad física parece ser protectora para la EII al reducir la inflamación sistémica. Si bien los episodios de muy alta intensidad desencadenan la inflamación sistémica, el ejercicio regular tiene acciones antiinflamatorias. El estilo de vida sedentario conduce a la expresión reducida de PGC-1a (coactivador del receptor gamma activado del proliferador de peroxisomas) que da como resultado un aumento de la respuesta inflamatoria sistémica. El PGC-1a reduce la actividad del factor nuclear-kB (NF-kB), que conduce a la producción de citoquinas proinflamatorias ${ }^{18}$. Varios estudios han demostrado el efecto protector del ejercicio $^{19,20}$. En un estudio prospectivo, el riesgo de desarrollar CU y EC en mujeres que realizaban actividad física fue de 8 y 6 eventos por 100.000 personas/año en comparación con 11 y 16 eventos por 100.000 personas/persona entre las mujeres sedentarias, respectivamente ${ }^{19}$.

\section{Vitamina D}

La vitamina $\mathrm{D}$, puede modificar la respuesta inmune innata, atenuando la transcripción de citoquinas proinflamatorias que se regulan positivamente en caso de estrés epitelial, además de mantener la integridad de las células epiteliales y mejorar la respuesta inmune innata en la mucosa ${ }^{21}$. Niveles elevados de vitamina $D$ han sido asociados de manera inversa con la EII ${ }^{22}$. En un estudio de cohorte prospectivo, personas con niveles adecuados de vitamina $\mathrm{D}(>30 \mathrm{ng} / \mathrm{ml})$ tuvieron una probabilidad significativamente menor de desarrollar EC y CU (OR 0,38; IC95\% 0,15-0,97 y OR 0,57; IC95\% 0,19-1,70, respectivamente $)^{23}$. En un estudio de 3.217 pacientes con EII, individuos con niveles séricos de vitamina $\mathrm{D}<20 \mathrm{ng} / \mathrm{ml}$ tenían mayor riesgo de cirugía y hospitalizaciones relacionadas con EII que aquellos con niveles $>30 \mathrm{ng} /$ $\mathrm{ml}$ (OR 1,76; IC95\% 1,24-2,51 y OR 2,07; IC95\% $1,59-2,68$, respectivamente $)^{24}$.

\section{Factores asociados a incremento del riesgo de EII}

\section{Calidad del sueño}

Estudios sugieren que el sueño y la alteración circadiana pueden afectar la permeabilidad intestinal, la translocación de endotoxinas bacterianas, la disbiosis intestinal y las citoquinas proinflamatorias $^{25}$.
En pacientes con EII en remisión, la mala calidad del sueño se asoció con un mayor riesgo de recaída de la enfermedad a los 6 meses en EC, no así en CU (OR 2; IC95\% 1,45-2,76 y OR 1,14; IC95\% 0,75-1,74, respectivamente $)^{26}$. Un estudio reciente, realizado con una polisomnografía que incluyó registro de saturación capilar, mostró que la desaturación bajo $90 \%$ fue más frecuente en pacientes con EII en remisión que en controles sanos, sugiriendo que la hipoxia podría tener un rol en la patogenia de la $\mathrm{EII}^{27}$. No se encontraron ensayos clínicos que evalúen si la mejora conductual o farmacológica de la calidad del sueño en la EII se asocia con mejores resultados de la enfermedad.

\section{Condiciones sanitarias/higiene}

En general, un alto nivel de higiene aumentaría el riesgo de EII. Una hipótesis es que la mejoría en las condiciones sanitarias es responsable de una disminución de la diversidad de la microbiota. Estudios han sugerido que el vivir en un entorno urbano y tener un menor número de hermanos podrían ser factores de riesgo para el desarrollo de $\mathrm{EII}^{28}$.

Por otra parte, la exposición a helmintos en la infancia demostró ser protectora con respecto al desarrollo de $\mathrm{EII}^{29}$, aunque otros no demostraron dicha asociación ${ }^{30}$. Un estudio realizado en ratones demostró que la infección con Echinococcus granulosus disminuyó la producción de óxido nítrico, factor de necrosis tumoral alfa (TNF- $\alpha$ ), óxido-nítrico-sintetasa inducible y de NF-kB en la mucosa colónica ${ }^{31}$. Por otro lado, una salud oral deficiente ${ }^{32}$ y el tener mascotas reduciría el riesgo de desarrollar $\mathrm{EII}^{33}$.

\section{Polución/Urbanización}

La exposición del intestino a los contaminantes puede ocurrir por inhalación de gases, eliminación mucociliar de material particulado de los pulmones y contaminación de las fuentes de agua y alimentos ${ }^{34}$. Estudios epidemiológicos mostraron que la EII es más prevalente en centros urbanos industrializados (RR 1,49 en EC y RR 1,63 en $\mathrm{CU})^{35}$. La exposición a partículas atmosféricas aumenta la permeabilidad intestinal, la respuesta inmune innata en el intestino, la expresión de citoquinas proinflamatorias y altera la microbiota ${ }^{36}$. Un estudio demostró que individuos residentes en regiones con concentraciones altas de dióxido nitroso y dióxido de azufre tenían más probabilida- 
des de desarrollar EII (OR 2,31; IC95\% 1,25-4,28 y OR 2,00; IC95\% 1,08-3,72, respectivamente $)^{34}$. Por otra parte, a mayor exposición a material particulado fino de peso molecular $<10$ micras existiría un menor riesgo de $\mathrm{EII}^{37}$.

\section{Obesidad}

La incidencia de EII está aumentando paralelamente con el sobrepeso y la obesidad. Aproximadamente $15-40 \%$ de los pacientes con EII tiene sobrepeso. De los diversos compartimentos de la grasa corporal, se sabe que la adiposidad visceral es la fracción metabólicamente activa, y podría ser más predictiva del riesgo de desarrollar EII que la obesidad general determinada por el índice de masa corporal (IMC). La obesidad se reconoce como un estado perpetuo de inflamación crónica, a través de un aumento de citoquinas proinflamatorias producidas por los adipocitos y la conversión de preadipocitos a macrófagos ${ }^{38}$. En cohortes prospectivas, la obesidad se asoció con el desarrollo de EC pero no de CU (OR 1,63, IC $95 \%, 1,07-2,50)^{39}$. Existen datos contradictorios sobre si la obesidad influye en la evolución de la EII. La obesidad visceral en pacientes con EC mostró un aumento en la probabilidad de cirugía $y$ de enfermedad penetrante ${ }^{40}$. Sin embargo, en un metaanálisis sobre el curso de la enfermedad en pacientes con EII, los resultados demostraron que pacientes obesos tenían una probabilidad menor de cirugía que los pacientes no obesos (RR 0,82; IC95\% 0,72-0,93). Esto podría explicarse por que un IMC más bajo podría ser simplemente el resultado de la progresión inflamatoria y que la obesidad sea un reflejo de una EII menos grave ${ }^{41}$.

\section{Antinflamatorios no esteroidales (AINE)}

Los AINE alteran la composición del moco al inhibir la secreción de bicarbonato y prostaglandinas e inhiben la secreción de fosfolípidos, alterando la permeabilidad intestinal.

Estudios han mostrado una asociación entre AINE y el riesgo de recaída en EII. Pacientes con EC con un uso de AINE $\geq 5$ veces al mes presentaron mayor riesgo de enfermedad activa en el seguimiento a 6 meses ( $23 \%$ versus $15 \%, p=0,04$ ). Sin embargo, no se observó ningún efecto en pacientes con CU (22\% versus $21 \%, \mathrm{p}=0,98)^{42}$. En un ensayo clínico se aleatorizaron pacientes con EII en remisión, un grupo recibió paracetamol y otro AINE convencionales durante 4 semanas. El grupo AINE tuvo una tasa de recaída de $17-28 \%$ dentro de 9 días y ninguno en el grupo con paracetamol $^{43}$. La inhibición selectiva de COX2 no se relacionó con la recaída de la EII ${ }^{44}$.

\section{Antibióticos y gastroenteritis}

Es conocido que el uso de antibióticos disminuye la diversidad de la microbiota aumentando el riesgo de EII ${ }^{45,46}$. El riesgo de desarrollar EC aumenta de forma dependiente de la dosis, principalmente cuando se usan antibióticos en el primer año de vida ${ }^{47}$.

Posterior a un cuadro de gastroenteritis infecciosa, el riesgo de desarrollar EII aumenta significativamente (RR 1,4, IC95\% 1,19-1,66), principalmente las infecciones entéricas como Escherichia coli adherente-invasivo, Salmonella, Campylobacter, especies de Mycobacterium avium $^{48,49}$. Esta asociación se puede explicar por la producción de IL-6, el bloqueo inespecífico de las células $\mathrm{T}$ reguladoras y la activación de la autodestrucción y la disbiosis ${ }^{50}$.

\section{Anticonceptivos orales}

Los anticonceptivos orales (ACO) se han asociado con un mayor riesgo de EII (OR 1,32; IC95\% 1,17-1,49) ${ }^{51}$. En la EC, el uso de una combinación de ACO con estrógeno y progestina se asoció con una mayor probabilidad de cirugía ${ }^{52}$, no así en $\mathrm{CU}^{53}$.

\section{Hipoxia}

En estudios en humanos, los niveles de IL6, IL1 y PCR aumentan en condiciones hipóxicas hipobáricas, como la altura ${ }^{54}$. Un estudio retrospectivo comparó la frecuencia de vuelos o viajes a regiones con una altitud mayor a $2.000 \mathrm{~m}$ sobre el nivel del mar, en pacientes con EII activos y en remisión, observando que $40,4 \%$ de los pacientes activos había estado a mayor de $2.000 \mathrm{~m}$ del mar versus $15,7 \%$ de los pacientes en remisión $(\mathrm{p}<0,005)^{55}$. Un pequeño ensayo piloto aleatorizado incluyó a 18 pacientes con $\mathrm{CU}$, observando que la oxigenoterapia hiperbárica es beneficiosa en la $\mathrm{CU}$ moderada-grave (remisión colónica al día 10 en $80 \%$ vs $25 \%, p=0,005)^{56}$.

\section{Estrés/factores anímicos}

El estrés, la ansiedad y la depresión han demostrado que empeora el curso de la $\mathrm{EII}^{57}$. Se desconoce si la comorbilidad psicológica es un 
verdadero factor de riesgo para el desarrollo de la EII, sin embargo, la EII empeora la depresión y se asocia con la resistencia al tratamiento ${ }^{57}$. Los pacientes con EII presentan una mayor incidencia de ansiedad y depresión ${ }^{58}$, ambas relacionadas con un aumento de las tasas de crisis ${ }^{59}$. La comorbilidad psicológica puede llevar a cambios en las conductas de salud, afectando la dieta, mal dormir, menor adherencia al tratamiento y aumento del tabaquismo ${ }^{57}$.

\section{Factores paradojales en la patogenia de la EII}

\section{Dieta}

Con la urbanización se han generado modificaciones en la dieta y, a su vez, en la composición de la microbiota intestinal, que podrían condicionar una respuesta inmune intestinal inadecuada y favorecer el desarrollo de EII ${ }^{60}$. El aumento de bacterias sulfurógenas, junto con una disminución de las bacterias protectoras, podrían alterar la homeostasis inmune local, aumentando la permeabilidad de la mucosa, lo que llevaría a una pérdida de tolerancia inmunológica ${ }^{61}$. En cuanto a los macronutrientes de la dieta, estudios han demostrado que las fibras vegetales son protectoras para la EII, sobre todo las frutas (OR: 0,69 IC95\% 0,49-0,96 y OR 0,57 IC95\% 0,44-0,74 para CU y $\mathrm{EC}$, respectivamente). En cuanto a las verduras, hubo asociación para $\mathrm{CU}$, pero no en $\mathrm{EC}^{62}$. Un posible mecanismo de protección asociado con la fibra dietética es la generación de ácidos grasos de cadena corta por las bacterias colónicas, que han demostrado poseer propiedades antiinflamatorias ${ }^{63}$.

La ingesta dietética de ácido graso poliinsaturado omega 3 (PUFA-3), que se encuentra en las grasas de los pescados, ha sido inversamente asociada al riesgo de CU, mientras que la dieta de PUFA-6, por ejemplo, la grasa de origen vacuno y la yema de huevo, se asocia con un mayor riesgo de $\mathrm{CU}^{64}$.

En cuanto a los emulsionantes (polisorbato 80, carboximetilcelulosa) que se usan para estabilizar sustancias con alto contenido en grasas, como los helados de crema, se ha visto que actúan directamente sobre la microbiota, aumentando el potencial proinflamatorio con aumento de las flagelinas bioactivas ${ }^{65} \mathrm{y}$ el riesgo de colitis ${ }^{66}$.

Por otro lado, el consumo de azúcares y pro- ductos con carbohidratos refinados y procesados puede ser un factor de riesgo para el desarrollo de $\mathrm{EII}^{67}$.

Respecto a los micronutrientes, algunos de ellos, como el zinc, desempeñan papeles importantes como coenzimas de metaloproteasas implicadas en el mantenimiento de la barrera epitelial intestinal. En un estudio de cohorte prospectivo, un mayor consumo de zinc ( $16 \mathrm{mg} /$ día) se asoció inversamente con el riesgo de EC (OR 0,63; IC95\% $0,43-0,93)^{68}$. Pacientes con niveles bajos de zinc tendrían un mayor riesgo de hospitalizaciones y cirugía, tanto en EC como en CU (OR 1,44; IC95\% 1,02-2,04 y OR 1,64; IC95\% 0,59-4,52, respectivamente) ${ }^{69}$.

\section{Apendicectomía}

La apendicectomía puede estar asociada significativamente con un mayor riesgo de EC (OR 1,61; IC95\% 1,28-2,2), principalmente durante el primer año posterior a la cirugía (OR 6,69; IC95\% $5,42-8,25)^{70}$. Por el contrario, el apéndice puede ser un órgano que favorecería el desarrollo de $\mathrm{CU}$, dado la mayor cantidad de linfocitos $\mathrm{T}$ y de células T CD4 CD69 de activación temprana ${ }^{71}$. La apendicectomía antes de los 20 años de edad es un factor protector de $\mathrm{CU}^{72}$.

En un estudio retrospectivo, la apendicectomía realizada después del diagnóstico de CU se asoció a mayor tasa de colectomía en su evolución (OR 2,2; IC95\% 1,1-4,5) ${ }^{73}$. Se esperan los resultados de un ensayo multicéntrico que incluye pacientes con CU en remisión, aleatorizados en grupo control con tratamiento de mantenimiento con 5-ASA sin apendicectomía y grupo con apendicectomía laparoscópica electiva más tratamiento de mantenimiento con 5-ASA. El objetivo es evaluar la eficacia a corto y mediano plazo de la apendicectomía para mantener la remisión en $\mathrm{CU}^{74}$.

\section{Tabaco}

Estudios han demostrado que el tabaquismo es protector para CU y perjudicial para EC. Entre ellos destacamos un metaanálisis que señala que el tabaquismo produciría una reducción del riesgo en CU (OR: 0,58, IC: 0,45-0,75), mientras que incrementaría el riesgo de EC (OR: 1,76 IC: 1,40$2,22)^{75}$. Contrario a lo anterior, solamente un estudio reciente prospectivo de casos y controles llegó a la conclusión de que no fumar resulta ser un factor protector tanto para $\mathrm{CU}$ como para 
$\mathrm{EC}^{76}$. Se requiere mayor evidencia para confirmar estos últimos resultados. En EC, el tabaco ha sido asociado a mayor riesgo de crisis, cirugía y falta de respuesta a tratamiento ${ }^{77}$.

Dentro de los componentes del cigarrillo, el monóxido de carbono inhibe la maduración de las células dendríticas y reduce la presentación de antígeno, como también la producción de citoquinas proinflamatorias y proliferación de linfocitos T efectores y estimula la producción de citoquinas antiinflamatorias ${ }^{77}$. La nicotina, por su parte, tendría un efecto inmunomodulador mediado por la activación de receptores nicotínicos $\alpha 7$ en macrófagos y células dendríticas, disminuyendo la producción de citoquinas proinflamatorias ${ }^{77}$.

El impacto paradojal del cigarrillo pareciera apuntar a un efecto sitio específico. Estudios en modelos murinos han demostrado que la exposición al cigarrillo exacerba el daño en intestino delgado $^{78}$, mientras que reduce la colitis ${ }^{79}$. Esto puede deberse a una llegada diferencial de los componentes del cigarrillo a los distintos tejidos. El material particulado es deglutido, alcanzando el intestino delgado, donde son absorbidos a medida que aumenta el $\mathrm{pH}$ hacia la zona distal, particularmente en el íleon, lo que explicaría el efecto en la $\mathrm{EC}^{77-79}$.

\section{Conclusión}

Ninguno de los factores ambientales por sí solo podría explicar el inicio y desarrollo de la EII. La combinación de factores genéticos, ambientales y una desregulación del sistema inmune es la hipótesis fisiopatológica de esta enfermedad. Descubrir cómo los factores ambientales influyen en la aparición de la EII y contribuyen a su patogénesis podría ayudar a reducir la enfermedad o su curso clínico. Las influencias ambientales podrían conducir a la identificación de nuevos objetivos terapéuticos y estrategias de tratamiento.

\section{Referencias}

1. de Souza HSP, Fiocchi C, Illiopoulos D. The IBD interactome: an integrated view of aetiology, pathogenesis and therapy. Nat Rev Gastroenterol Hepatol 2017; 14: 739-49.

2. Ng SC, Shi HY, Hamidi N, Underwood FE, Tang W, Benchimol EI, et al. Worldwide incidence and prevalen- ce of inflammatory bowel disease in the 21 st century: a systematic review of population-based studies. Lancet 2018; 23; 390: 2769-78.

3. Simian D, Fluxá D, Flores L, Lubascher J, Ibáñez P, Figueroa $\mathrm{C}$, et al. Inflammatory Bowel Disease: a prospective study of 716 local Chilean patients. World J Gastroenterol 2016; 22: 5267-75.

4. Kaplan GG. The global burden of IBD: from 2015 to 2025. Nat Rev Gastroenterol Hepatol 2015; 12: 720-7.

5. Rogler G, Vavricka S. Exposome in IBD: Recent insights in environmental factors that influence the onset and course of IBD. Inflamm Bowel Dis 2015; 21: 400-8.

6. Kunz C, Rudloff S, Baier W, Klein N, Strobel S. Oligosaccharides in human milk: structural, functional, and metabolic aspects. Annu Rev Nutr 2000; 20: 699-722.

7. Klement E, Cohen RV, Boxman J, Joseph A, Reif S. Breastfeeding and risk of inflammatory bowel disease: a systematic review with metaanalysis. Am J Clin Nutr 2004; 80: 1342-52.

8. Xu L, Lochhead P, Ko Y, Claggett B, Leong RW, Ananthakrishnan AN. Systematic review with meta-analysis: breastfeeding and the risk of Crohn's disease and ulcerative colitis. Aliment Pharmacol Ther 2017; 46: 780-9.

9. Castaño-Rodríguez N, Kaakoush NO, Lee WS, Mitchell HM. Dual role of Helicobacter and Campylobacter species in IBD: a systematic review and meta-analysis. Gut 2017; 66: 235-49.

10. Rad R, Brenner L, Bauer S, Schwendy S, Layland L, da Costa CP, et al. CD25+/Foxp3+ T cells regulate gastric inflammation and Helicobacter pylori colonization in vivo. Gastroenterology 2006; 131: 525-37.

11. Abraham BP, Quigley EMM. Probiotics in Inflammatory Bowel Disease. Gastroenterol Clin North Am 2017; 46: 769-82.

12. Groeger D, O’Mahony L, Murphy EF, Bourke JF, Dinan TG, Kiely B, et al. Bifidobacterium infantis 35624 modulates host inflammatory processes beyond the gut. Gut Microbes 2013; 4: 325-39.

13. Krag $A$, Munkholm $P$, Israelsen $H$, von Ryberg $B$, Andersen KK, Bendtsen F. Profermin is efficacious in patients with active ulcerative colitis - A randomized controlled trial. Inflamm Bowel Dis 2013; 19: 2584-92.

14. Dong J, Teng G, Wei T, Gao W, Wang H. Methodological quality assessment of meta-analyses and systematic reviews of probiotics in inflammatory bowel disease and pouchitis. PLoS One 2016; 11: e0168785.

15. Shen J, Zuo ZX, Mao AP. Effect of probiotics on inducing remission and maintaining therapy in ulcerative colitis, Crohn's disease, and pouchitis: meta-analysis of randomized controlled trials. Inflamm Bowel Dis 2014; 20: 21-35. 
16. Taekil Eom, Yong Sung Kim, Chang Hwan Choi, Michael J. Sadowsky, Tatsuya Unno. Current understanding of microbiota and dietary therapies for treating inflammatory bowel disease. J Microbiol 2018; 56 (3): 189-98.

17. Laurell A, Sjöberg K. Prebiotics and synbiotics in ulcerative colitis. Scand J Gastroenterol 2017; 52 (4): 477-85.

18. Bilski J, Mazur-Bialy A, Brzozowski B, Magierowski M, Zahradnik-Bilska J, Wojcik D, et al. Can exercise affect the course of inflammatory bowel disease? Experimental and clinical evidence. Pharmacological Rep 2016; 68: 827-36.

19. Khalili H, Ananthakrishnan AN, Konijeti GG, Liao X, Higuchi LM, Fuchs CS, et al. Physical activity and risk of inflammatory bowel disease: prospective study from the Nurses' Health Study cohorts. BMJ 2013; 347: f6633.

20. Jones PD, Kappelman MD, Martin CF, Chen W, Sandler RS, Long MD. Exercise decreases risk of future active disease in patients with inflammatory bowel disease in remission. Inflamm Bowel Dis 2015; 21: 1063-71.

21. Abdo J, Rai V, Agrawai DK. Interplay of Immunity and Vitamin D: Interactions and Implications with Current IBD Therapy. Curr Med Chem 2017; 24: 852-67.

22. Jaime F, Riutort MC, Alvarez-Lobos M, Hoyos-Bachiloglu R, Camargo CA Jr, Borzutzky A. Solar radiation is inversely associated with inflammatory bowel disease admissions. Scand J Gastroenterol 2017; 52 (6-7): 730-7.

23. Ananthakrishnan AN, Khalilli H, Higuchi LM, Bao Y, Korzenik JR, Richter JM, et al. Higher predicted vitamin D status is associated with reduced risk of Crohn's disease. Gastroenterology 2012; 142: 482-9.

24. Ananthakrishnan AN, Cagan A, Gainer VS, Cai T, Cheng SC, Savova G, et al. Normalization of plasma 25-hydroxy vitamin $\mathrm{D}$ is associated with reduced risk of surgery in Crohn's disease. Inflamm Bowel Dis 2013; 19: 1921-7.

25. Swanson GR, Burgess HJ. Sleep and Circadian Hygiene and Inflammatory Bowel Disease. Gastroenterol Clin North Am 2017; 46: 881-93.

26. Ananthakrishnan AN, Long MD, Martin CF, Sandler RS, Kappelman MD. Sleep disturbance and risk of active disease in patients with Crohn's disease and ulcerative colitis. Clin Gastroenterol Hepatol 2013; 11: 965-71.

27. Bar-Gil Shitrit A, Chen-Shuali C, Adar T, Koslowsky B, Shteingart S, Paz K, et al. Sleep Disturbances Can Be Prospectively Observed in Patients with an Inactive Inflammatory Bowel Disease. Dig Dis Sci 2018; 63: 2992-7.

28. Cholapranee A, Ananthakrishnan AN. Environmental hygiene and risk of inflammatory bowel diseases: a systematic review and meta-analysis. Inflamm Bowel Dis 2016; 22: 2191-9.
29. Chu KM, Watermeyer G, Shelly L, Janssen J, May TD, Brink K, et al. Childhood helminth exposure is protective against inflammatory bowel disease: a case control study in South Africa. Inflamm Bowel Dis 2013; 19: 614-20.

30. Bager P, Vinkel Hansen A, Wohlfahrt J, Melbye M. Helminth infection does not reduce risk for chronic inflammatory disease in a population-based cohort study. Gastroenterology 2012; 142: 55-62.

31. Khelifi L, Soufli L, Labsi M, Touil-Boukoffa C. Immune-protective effect of echinococcosis on colitis experimental model is dependent of dowm regulation of TNF alfa and NO production. Acta Trop 2017; 166: 7-15.

32. Yin W, Ludvigsson JF, Liu Z, Roosaar A, Axeli T, Ye W. Inverse Association Between Poor Oral Health and Inflammatory Bowel Diseases. Clin Gastroenterol Hepatol 2017; 15: 525-31.

33. Ng SC, Tang W, Leong RW, Chen M, Ko Y, Studd $\mathrm{C}$, et al. Environmental risk factors in inflammatory bowel disease: a population-based case-control study in Asia-Pacific. Gut 2015; 64 (7): 1063-71.

34. Kaplan GG, Hubbard J, Korzenik J, Sands BE, Panaccione R, Ghosh S, et al. The inflammatory bowel diseases and ambient air pollution: a novel association. Am J Gastroenterol 2010; 105: 2412-9.

35. Juillerat P, Pittet V, Bulliard JL, Guessous I, Antonino AT, Mottet C, et al. Prevalence of inflammatory bowel disease in the Canton of Vaud (Switzerland): a population-based cohort study. J. Crohns Colitis 2008; 2: 131-41.

36. Kish L, Hotte N, Kaplan GG, Vincent R, Tso R, Gänzle $\mathrm{M}$, et al. Environmental particulate matter induces murine intestinal inflammatory responses and alters the gut microbiome. Plos One 2013; 8: e62220.

37. Opstelten JL, Beelen RMJ, Leenders M, Hoek G, Brunekreef B, van Schaik FDM, et al. Exposure to ambient air pollution and the risk of inflammatory bowel disease: A European Nested Case-Control Study. Dig Dis Sci 2016; 61: 2963-71.

38. Singh S, Dulai PS, Zarrinpar A, Ramamoorthy S, Sandborn WJ. Obesity in IBD: epidemiology, pathogenesis, disease course and treatment outcomes. Nat Rev Gastroenterol Hepatol 2017; 14: 110-21.

39. Khalili H, Ananthakrishnan AN, Konijeti GG, Higuchi LM, Fuchs CS, Richter JM, et al. Measures of obesity and risk of Crohn's disease and ulcerative colitis. Inflamm Bowel Dis 2015; 21: 361-8.

40. Van Der Sloot KW, Joshi AD, Bellavance DR, Gilpin KK, Stewart KO, Lochhead P, et al. Visceral adiposity, genetic susceptibility, and risk of complications among individuals with Crohn's disease. Inflamm Bowel Dis 2017; 23: 82-8. 
41. Hu Q, Ren J, Li G, Wu X, Li J. The Impact of obesity on the clinical course of Inflammatory Bowel Disease: A Meta-Analysis. Med Sci Monit 2017; 23: 2599-606.

42. Long MD, Kapplelman MD, Martin CF, Chen W, Anton $\mathrm{K}$, Sandier RS. Role of nonsteroidal anti-inflammatory drugs in exacerbations of inflammatory bowel disease. J Clin Gastroenterol 2016; 50: 152-6.

43. Takeuchi K, Smale S, Premchand P, Maiden L, Sherwood R, Bjornsson E, et al. Prevalence and mechanism of nonsteroidal anti-inflammatory drug-induced clinical relapse in patients with inflammatory bowel disease. Clin Gastroenterol Hepatol 2006; 4: 196-202.

44. Sandborn WJ, Stenson WF, Brynskov J, Lorenz RG, Steidle GM, Robbins JL, et al. Safety of celecoxib in patients with ulcerative colitis in remission: a randomized, placebo-controlled, pilot study. Clin Gastroenterol Hepatol 2006; 4: 203-11.

45. Ananthakrishnan AN, Bernstein CN, Iliopoulos D, Macpherson A, Neurath MF, Ali RAR, et al. Environmental triggers in IBD: a review of progress and evidence. Nat Rev Gastroenterolol Hepatol 2018; 15: 39-49.

46. Ungaro R, Bernstein CN, Gearry R, Hviid A, Kolho Kl, Kronman MP, et al. Antibiotics associated with increased risk of new-onset Crohn's disease but not ulcerative colitis: a meta-analysis. Am J Gastroenterol 2014; 109: 1728-38.

47. Kronman MP, Zaoutis TE, Haynes K, Feng R, Coffin SE. Antibiotic exposure and IBD development among children: a population-based cohort study. Pediatrics 2012; 130: e794-803.

48. Jess T, Simonsen J, Nielsen NM, Jørgensen KT, Bager P, Ethelberg S, et al. Enteric Salmonella or Campylobacter infections and the risk of inflammatory bowel disease. Gut 2011; 60: 318-24.

49. Naser SA, Sagramsingh SR, Naser AS, Thanigachalam S. Mycobacterium avium subspecies paratuberculosis causes Crohn's disease in some inflammatory bowel disease patients. World J Gastroenterol 2014; 20: 7403-15.

50. Porter CK, Tribble DR, Aliaga PA, Halvorson HA, Riddle MS. Infectious gastroenteritis and risk of developing inflammatory bowel disease. Gastroenterology 2008; 135: 781-6.

51. Ortizo R, Lee SY, Nguyen ET, Jamal MM, Bechtold MM, Nguyen DL. Exposure to oral contraceptives increases the risk for development of inflammatory bowel disease: a meta-analysis of case-controlled and cohort studies. Eur J Gastroenterolo Hepatol 2017; 29: 1064-70.

52. Khalili H, Granath F, Smedby KE, Ekbon A, Neovius $\mathrm{M}$, Chan AT, et al. Association between long-term oral contraceptive use and risk of Crohn's disease com- pli- cations in a nationwide study. Gastroenterology 2016; 150: 1561-7.

53. Khalili H, Neovius M, Ekbom A, Ludvigsson JF, Askling J, Chan AT, et al. Oral contraceptive use and risk of ulcerative colitis progression: a nationwide study. Am J Gastroenterol 2016; 111: 1614-20.

54. Hartmann G, Tschöp M, Fischer R, Bidlingmaier C, Riepl R, Tschöp K, et al. High altitude increases circulating interleukin-6, interleukin-1 receptor antagonist and C-reactive protein. Cytokine 2000; 12: 246-52.

55. Vavricka SR, Rogler G, Maetzler S, Misselwitz B, Safroneeva E, Frei P, et al. High altitude journeys and flights are associated with an increased risk of flares in inflammatory bowel disease patients. J Crohns Colitis 2014; 8: 191-9.

56. Dulai P, Buckey Jr J, Raffals L, Swoger J, Claus P, O’Toole $\mathrm{K}$, et al. Hyperbaric oxygen therapy is safe and effective for hospitalized ulcerative colitis patients suffering from moderate-severe flares: a multi-center, randomized, double-blind, sham-controlled trial. Gastroenterology 2017; 152: S198.

57. Bernstein CN. Psycological stress and depression: risk factors for IBD? Dig Dis 2016; 34: 58-63.

58. Fuller-Thomson E, Sulman J. Depression and inflammatory bowel disease: finding from two nationally representative Canadian surveys. Inflamm Bowel Dis 2006; 12: 697-707.

59. Mittermaier C, Dejaco C, Waldhoer T, Oefferlbauer-Ernst A, Miehsler W, Beier M, et al. Impact of depressive mood on relapse in patients with inflammatory bowel disease: a prospective 18-month follow-up study. Psychosom Med 2004; 66: 79-84.

60. Brown K, DeCoffe D, Molcan E, Gibson DL. Diet-induced dysbiosis of the intestinal microbiota and the effects on immunity and disease. Nutrients 2012; 4: 1095-119.

61. Lewis JD, Abreu MT. Diet as a trigger or therapy for inflammatory bowel diseases. Gastroenterology 2017; 152: 398-414.

62. Li F, Liu X, Wang W, Zhang D. Consumption of vegetables and fruit and the risk of inflammatory bowel disease: a meta-analysis. Eur J Gastroenterol Hepatol 2015; 27: 623-30.

63. Vinolo MA, Rodrigues HG, Renato T., Nachbar RT, Curi R. Regulation of inflammation by short chain fatty acids. Nutrients 2011; 3: 858-76.

64. Ananthakrishnan AN, Khalili H, Konijeti GG, Higuchi LM, de Silva P, Fuchs CS, et al. Long-term intake of dietary fat and risk of ulcerative colitis and Crohn's disease. Gut 2014; 63: 776-84.

65. Chassaing B, Van de Wiele T, De Bodt J, Marzorati M, Gewirtz AT. Dietary emulsifiers directly alter human 
microbiota composition and gene expression ex vivo potentiating intestinal inflammation. Gut 2017; 66: 1414-27.

66. Chassaing B, Koren O, Goodrich JK, Poole AC, Srinivasan S, Ley RE, et al. Dietary emulsifiers impact the mouse gut microbiota promoting colitis and metabolic syndrome. Nature 2015; 519: 92-6.

67. Hansen TS, Jess T, Vind I, Elkjaer m, Nielsen mf, Gamborg $\mathrm{M}$, et al. Environmental factors in inflammatory bowel disease: a case-control study based on a Danish inception cohort. J. Crohns Colitis 2011; 5: 577-84.

68. Ananthakrishnan AN, Khalilli H, Song M, Higuchi LM, Richter JM, Chan AT. Zinc intake and risk of Crohn's disease and ulcerative colitis: a prospective cohort study. Int J Epidemiol 2015; 44: 1995-2005.

69. Silva S, Rubin DT, Gulotta G, Wroblewski K, Pekow J. Zinc deficiency is associated with poor clinical outcomes in patients with inflammatory bowel disease. Inflamm Bowel Dis. 2017; 23: 152-7.

70. Kaplan GG, Jackson T, Sands BE, Frisch M, Andersson RE, Korzenik J. The risk of developing Crohn's disease after an appendectomy: a meta-analysis. Am J Gastroenterol 2008; 103: 2925-31.

71. Matsushita M, Uchida K, Okazaki K. Role of the appendix in the pathogenesis of ulcerative colitis. Inflammopharmacology 2007; 15: 154-7.

72. Andersson RE, Olaison G, Tysk C, Ekbom A. Appendectomy and protection against ulcerative colitis. $\mathrm{N}$ Engl J Med 2001; 344: 808-14.
73. Parian A, Limketkai B, Koh J, Brant SR, Bitton A, Cho $\mathrm{JH}$, et al. Appendectomy does not decrease the risk of future colectomy in UC: results from a large cohort and meta-analysis. Gut 2017; 66: 1390-7.

74. Gardenbroek TJ, Pinkney TD, Sahami S, Morton DG, Buskens Cj, Ponsioen CY, et al. The ACCURE- trial: the effect of appendectomy on the clinical course of ulcerative colitis, a randomised international multicenter trial (NTR2883) BMC Surg 2015; 15: 30.

75. Mahid SS, Minor KS, Soto RE, Homung CA, Galandiuk S. Smoking and inflammatory bowel disease: a meta-analysis. Mayo Clin Proc 2006; 81: 1462-71.

76. Salih A, Widbom L, Hultdin J, Karling P. Smoking is associated with risk for developing inflammatory bowel disease including late onset ulcerative colitis: a prospective study. Scand J Gastroenterolol 2018; 53: 173-8.

77. Berkowitz L, Schultz BM, Salazar GA, Pardo-Roa C, Sebastián VP, Álvarez-Lobos MM, et al. Impact of Cigarette Smoking on the Gastrointestinal Tract Inflammation: Opposing Effects in Crohn's Disease and Ulcerative Colitis. Front Immunol 2018; 9: 74.

78. Zuo L, Li Y, Wang H, Wu R, Zhu W, Zhang W, et al. Cigarette smoking is associated with intestinal barrier dysfunction in the small intestine but not in the large intestine of mice. J Crohns Colitis 2014; 8: 1710-22.

79. Eliakim R, Fan QX, Babyatsky MW. Chronic nicotine administration differentially alters jejunal and colonic inflammation in interleukin-10 deficient mice. Eur J Gastroenterol Hepatol 2002; 14: 607-14. 